\title{
A user-centred approach to design Transport Interchange Hubs (TIH): A discussion illustrated by a case study in the Russian Arctic
}

\author{
Clarice Bleil de Souza ${ }^{1 a}$ ‘[0000-0001-7823-1202]’, Ilya V. Dunichkin² ‘[0000-0001-9372-0741]’, Ca- \\ milla Pezzica ${ }^{1 b}$ '[[0000-0002-0512-7591]' \\ ${ }^{1}$ Welsh School of Architecture - Cardiff University - Bute building, King Edward VII Ave- \\ nue, CF10 3NB, Cardiff - UK \\ ${ }^{2}$ Moscow State University of Civil Engineering, Yaroslavskoye Sh. 26, 129337, Moscow, Rus- \\ sia \\ 1aBleildesouzac@cardiff.ac.uk, ${ }^{2}$ ecse@bk.ru \\ 1bPezzicac@cardiff.ac.uk
}

\begin{abstract}
This paper proposes a user-centred approach to design Transport Interchange Hubs (TIH). It is based on a literature review of existing information related to TIHs outside the domain of transport engineering, so the focus is on the building and the usage of it by its main customers: the travellers. A literature review is used to extract high level information on travellers' needs, technical and functional requirements of TIHs, constraints and design parameters. A product development approach is used to classify and combine this data so a proper set of design specifications to better address users' needs is proposed. A case study in the Russian Arctic is presented to illustrate the discussion due to the complexity of needs, requirements and parameters involved in designing TIHs in extreme climates. A proof of concept, using Axiomatic Design, to develop design specifications and manage constraints is applied and discussed considering how different types of simulation tools, essential to assess performance of complex buildings, can be integrated to the design process. Robust specifications, despite being part of parametric design methods, are underexplored in the architecture design domain. This work intends to provide a contribution to the body of knowledge in this area opening avenues for further research in how to define common design targets and objectives for different stakeholders as well as to manage the collaborative work of consultants involved in designing complex buildings.
\end{abstract}

Keywords: Decision-making in design, Transport Interchange Hubs (TIH), Russian Arctic, Sustainable urban development.

\section{Introduction}

Transport Interchange Hubs (TIH) are points of exchange for people and/or cargo between different modes of transport and /or different types of vehicles. They "play a key 
role as part of public transport networks, facilitating the links between public transportation modes" [1]. Efficient TIHs can promote urban integration, be time savers, improve operational business models, and reduce the use of cars therefore contributing to diminish carbon emission addressing issues related to climate change. They are strategically important to many countries. In the EU, they are part of a strategic agenda which includes "the integration of a European high-speed rail network, the shift of road to freight transport over $300 \mathrm{~km}$ to other modes (i.e. rail or waterborne) and the connection of all core airports to the rail network." [2]. In China, they form part of the government economic and social development plan [3-5]. In Russia, they form part of the Strategy of Development of the Arctic Zone (Northern Sea Route) focused on the exploitation of mineral resources and the development of infrastructure around them [6].

As part of countries strategic agendas, TIH has been the topic of many research projects. In the EU, one can find at least five recent projects involving TIHs: HERMES [7]; City HUB [8]; NODES [9]; Alliance (2018) [10] and the recent VitalNodes [11]. In Russia, the focus is on modernizing transport network and infra-structure in the Arctic zone through the design, development and refurbishment of various sizes of TIH accommodating the peculiarities of migration flow in the Northern area. The average mobility index for the population of the Arctic is 9.8\%, meaning 1 in 10 people either move in or out of the region [12]. Mobility usually involves long distance flows, i.e. flows to long distant connections, normally to and from territories outside the Arctic. This has an impact in transport infra-structure, meaning TIH must be adaptable and flexible to cope with cities' 'pulsating' populations [12]

TIHs can be viewed as complex systems because of the demands related to function, cost, quality and sustainability involved in their designs. Beyond having to accommodate different transport functions, they are a technologically driven and market-oriented apparatus difficult to design, costly to build, operate and maintain. The problem gets even more complicated if flexibility and adaptability requirements are part of their design and operation agendas and they have to be built on harsh climatic zones as in the case of TIHs in the Russian Arctic.

Despite a significant recent number of EU projects related to TIHs as well as some major Chinese initiatives reported in the literature [4, 5 and 13 , to cite a few], systematic knowledge related to the design of TIHs remains unaddressed. The literature seems vast on the domain of transport engineering, but it is scarcely populated with a set of frameworks, methods, models and criteria to analyse and evaluate performances of the terminals in relation to building functioning and their relationships with the city (especially at the neighbourhood scale), user satisfaction and environmental concerns. Despite the recent focus on TIH customers' (travellers') satisfaction found in the literature (see section 2 below), to the best of the author's knowledge there are no clear and robust methodologies to develop design specifications to fulfil their needs.

This paper proposes a user-centred approach to develop a robust set of specifications for the design of TIH buildings. It uses Axiomatic Design (AD), a methodological approach from product design, to start listing and discussing travellers' needs, technical and functional requirements, constraints and design parameters involved in the design of TIHs. It also borrows AD assessment methods to address how different types of building performance simulations can be better integrated through the design process 
so design proposals can be assessed at different stages and design specifications amended and revisited if necessary. The user-centred approach is proposed as a proof of concept to illustrate the importance of developing a robust set of design specifications to attend the most important TIH user, its travel customer. Robust design specifications, despite being part of parametric design methods, are underexplored in the architecture design domain. The authors see them as a starting point to effectively reconcile design targets and objectives of different stakeholders as well as to manage the work of different consultants involved in designing these types of complex buildings.

\section{Customers' needs - the state of the art}

Most of the recent literature about TIH focuses on customer satisfaction and overall travel experience, possibly because studies of this type have been rare until around 2015. Hernandez et al [14] conducted a survey to collect travellers' data about various aspects of TIH and run a classification and regression tree model together with an important-performance analysis model to identify strengths and weaknesses on travellers' perceptions of a TIH in Spain. A similar work is proposed by [15], who produce a decision tree and apply descriptive and inferential statistics to surveys with customers to investigate user perception and satisfaction with quality of service provided by a TIH in Riga. Monzon et al [16] report, from surveys with different stakeholders including customers, the influence of nine basic parameters in the overall quality of five different terminals. Average scores for these parameters are calculated based on the findings so weaknesses and strengths of different terminals can be identified. Hickman et al [13] compare and contrast customer's surveys from three different Chinese terminals with actually what they deliver in order to calculate a 'disgruntlement level' for these terminals based on a hierarchy of customers' dissatisfaction indicators. Bryniarska et al [17] propose a set of generic criteria to assess the quality of TIH buildings and surveyed travellers' in relation to the importance of distance, quality of infra-structure and information also aiming to rank customers' needs to derive a weighting system to enable assessing the quality of interchanges using multi-criteria evaluation. Liu et al [5] and Li et al [4] use surveys with customers to set up models to investigate different accessibility scenarios to TIH generated through multi-criteria evaluation, focusing on reducing travelling time or gauging easiness of travelling, respectively.

Some important characteristics can be identified from these studies. The first one is the trend to focus on rankings and attributing scores to customers' needs so multi-criteria evaluation assessments can be undertaken with properly justifiable weighting. The second one is all studies display a set of overarching criteria behind travellers' needs and aspirations similarly to what is proposed by [18] and recommended by Netherlands, British and other EU railway bodies. Finally, the aim of these studies is centred in the assessment of customers' needs to gauge the quality of existing terminals rather than investigating how these needs can be translated into design specifications. As a result, the translation of these needs into design guidelines tend to be presented as gigantic and unstructured lists of requirements with mixed information. These lists are not easily manageable nor suitable to be readily used by building designers, i.e. they need to be 
translated into a set of useful design specifications with appropriate hierarchies and relationships to be directly applied in project work. This work illustrates a proof of concept about how information from the literature can be translated into a set of useful design specifications for TIH buildings by adopting an AD approach.

\section{Methodology: Adopting a product design approach}

The idea of using product design methods in architecture is not new. Marchesi and Matt [19] present a literature review on transferring product design methods, specifically Axiomatic Design (AD) to building design on the basis that AD would provide a more comprehensive analytical framework to problem specification which is extremely valuable in the design of complex buildings used by the general public as well as in projects in which many different types of consultants are involved. Besides that, a product design approach also has formal ways to assess project outcomes through targets, evaluation and assessment methods and procedures which can potentially contribute to 'extend' the assessment of architectural design solutions, facilitating the integration of simulation tools through the design process.

Developed by Nam and Suh at the MIT, AD provides a design specification model based on "principles of functional independence and complexity minimization [in which] problem and solutions are systematically specified in parallel, moving down along the hierarchy and design decisions are made in an explicit way maintaining data." [19]. The specification model is based on four different domains: the Customer domain in which customers' needs and aspirations are specified; the functional domain in which functional design requirements are specified; the Physical domain in which design parameters to meet requirements are specified and the Process domain which is concerned with the manufacturing of the parameters specified in the Physical domain [20]. During this mapping or translation process the designer needs to follow two Axioms: the 'Independence Axiom' and the 'Information Axiom'. The first is related to the independence of functional requirements, i.e. each functional requirement needs to be fulfilled independently of each other. The second is related to level of information of the design content, which must be kept to a minimum. This suggests "that physical integration is desirable to reduce the information content if the functional independence can be maintained" [20].

To the best of the authors' knowledge, there is no example of applying AD to the design of TIHs. The closest reference is [21], which applies AD to the design of an airport passenger's terminal. This paper borrows the structure of AD, to translate Customers' needs into Functional Requirements and subsequently Design Parameters, to develop a set of design specifications to better accommodate TIH travellers' needs since the early design stages (Fig. 1). Travellers' needs affect the perceived quality of the terminal and the transport services provided by it as well as the ability of this building to act as an economic hub for urban sustainable development. All these have an impact in the design of the building and its insertion into a given neighbourhood or city. Other stakeholders involved in the design and use of $\mathrm{TIH}$, for instance business managers, transport operators, public authorities etc. are not part of this study. However, important 
information referring to terminal capacity and operation as well as building standards and regulations are used as boundary conditions to this design problem and expressed as Input Constraints.

Input Constraints (ICs) are an imposed part of design specifications [20] and in this paper will be treated constraints related to: the existing infra-structure affecting the placement of the terminal in a given neighbourhood (IC1); the health and safety and fire safety regulations, transport safety standards, etc. (IC2); the extreme climatic conditions and restrictions in building on the permafrost (IC3) defined by (REF). IC1 and IC2 will not be explored in detail in this paper, as IC1 is site dependent and IC2 would mainly involve complying with regulations to whatever design specification is proposed. It would vary country by country and make the whole paper extremely long and prescriptive, diverging attention from the discussion on functional requirements and design parameters.

Besides the aforementioned constraints, the work addresses and discusses: (i) Travellers' Needs and Aspirations (TNAs), mainly using the Customers' Pyramid proposed by [18] together with further relevant information from the literature [13-17]; (ii) Functional Requirements (FRs), by translating Travellers' Needs and Aspirations into a set of functional specifications considering a 'solution neutral' environment and; (iii) Design Parameters (DPs) discussing and defining the physical variables to satisfy each FR individually listed. The work does not go beyond two levels of specification in terms of most of the FRs as it is the intention it remains solution neutral (unbiased) and can be used as a starting point to rethink the design of terminal buildings adopting a customer/ traveller perspective (Fig 1).

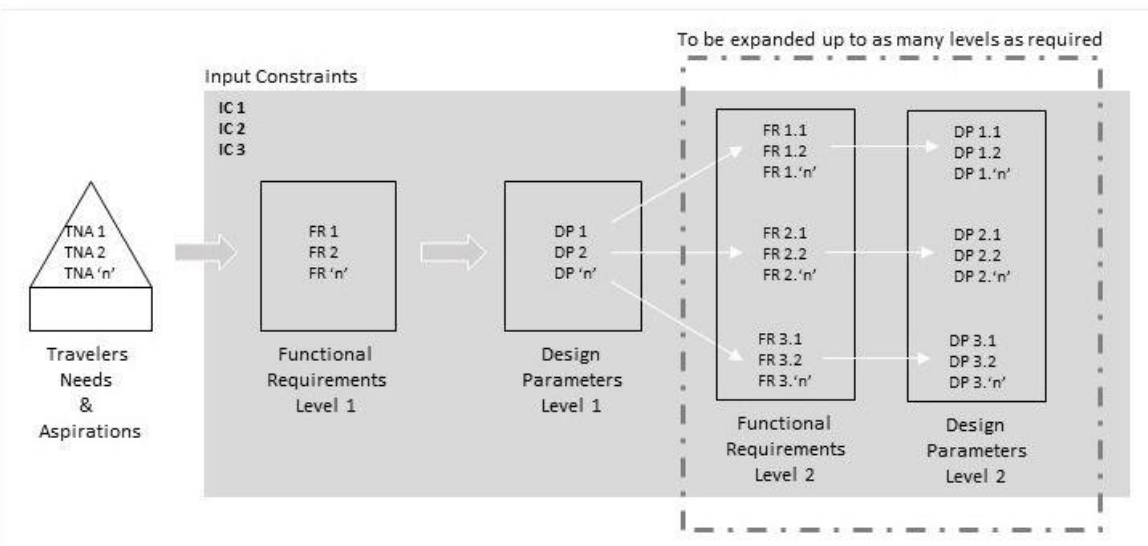

Fig. 1. Applying the Axiomatic Design structure to the design of TIH

Section 4 illustrates how the design problem decomposition is structured and how the first Axiom is applied. It starts by proposing how customers' needs can be translated into FRs and DPs as well as how ICs are imposed. An illustration of applying Axiom 1 is displayed in the second level of design specifications through three design matrices. These matrices are mathematical expressions of how FRs and DPs are related to each other. Design goals are defined as an FR vector whereas "the set of design parameters 
in the physical domain that has been chosen to satisfy the FRs constitutes the DP vector." [20]. If functional requirements are independent from each other, the matrix is diagonal and the design is called uncoupled. This is an ideal situation because it means each FR can be independently satisfied by a single DP enabling the design process to be fully controlled, facilitating manufacturing, maintenance and replacement of parts. If the matrix is triangular, "the independence of FRs can be guaranteed if and only if the DPs are determined in a proper sequence" [20]. This situation is called decoupled design and is also considered an acceptable solution because the clear hierarchy of FRs still enables the design process to be fully controlled as well as its manufacturing and maintenance. When the matrix has any other shape, either the design is redundant (having more parts then needed) or is out of control, meaning every time a parameter is changed the propagation of changes reaches different FRs and therefore different DPs.

Section 5 focuses on proposing how to meet the second Axiom in building design as well as how to trigger the zigzag process. The Second Axiom relates design success with complexity of information. The latter is defined in relation to the tolerance provided by each DP to fulfil required tolerances for each FR. This "probability of success can be computed by specifying the design range for the FR and by determining the system range that the proposed design can provide to satisfy the FR" [20]. The authors believe that assessing compatibility between probability ranges of FRs and DPs in building design calls for the use of building performance simulation. This part of the paper therefore discusses how different types of simulations can be better integrated throughout the design process from the early stages of design specifications. Since simulation results have an impact in gauging how proposed DPs affect each other, these can also be used to trigger the zigzag process in which FRs and DPs assessed at lower level specifications inform the correctness of dependencies predicted at the upper levels, enabling them to be changes and the whole specification process to be reassessed.

\section{Design(ing) specifications for TIH buildings}

Van Hagen and De Bruyn [18] provide an upper level comprehensive pyramid of travellers' needs and/or aspirations to be addressed (Fig. 2), towards which most of the existing literature on customers' needs seems to converge (see section 2 of this paper). Needs and aspirations at each level of this pyramid are related to: the transport service provided, the staff, as well as the place where the service is provided-i.e. the building. In general, as one goes down in the pyramid the weighting of the services in fulfilling needs and aspirations of travellers increases and the building mainly acts as a 'background' to enable services and people to properly operate in the fulfilment of these needs.

Fulfilling needs at the bottom of the pyramid mean the building mainly needs to comply with a set of regulations which impose conditions for transport service operation to happen in a safe and secure way. These conditions are expressed as a set of building design constraints (health and safety, fire safety, transport operation, etc.) defined in this paper as IC2, which would be imposed at different levels of specification for FRs and DPs. However, when considering building TIHs in the Arctic, 'Safety and 
Security' needs must be extended to include constraints related to building in extreme climatic conditions. These constraints have clear implications in building layout and building envelope design (IC3) and can potentially affect the design specification from its very beginning.

Major constraints related to extreme climatic conditions and restrictions in building on the permafrost (IC3) include requirements for almost all spaces to be enclosed. As part of this constraint indoor air temperatures need to be controlled at different ranges according to the following types of space usage: spaces of permanence such as sitting area, between $18^{\circ} \mathrm{C}$ and $24^{\circ} \mathrm{C}$; circulation spaces, between $0^{\circ} \mathrm{C}$ and $5^{\circ} \mathrm{C}$ and platform areas between $-15^{\circ} \mathrm{C}$ to $0^{\circ} \mathrm{C}$ [22]. Besides that, building on the permafrost implies having compact and large footprint buildings with prohibitive costs of using underground spaces, deep pile foundations and concentrated building utility entry points [23].

The second layer of the pyramid, 'Reliability' is again very dependent on transport service provision. However, the role of the building changes to the one of an 'enabler', meaning it has to provide adequate capacity, and potentially flexibility and adaptability, to hold and deliver the different types of transport services assigned to it. Transport and traffic recommendation values and variables are applied as a set of design constraints to guarantee adequate service availability, therefore fulfilling travellers' reliability needs. These constraints are also part of IC2 as they are defined by transport operation guidelines which include a series of safety parameters.

The remaining layers of the pyramid have large implications on the design of the terminal building, particularly in decisions related to its layout and enclosure, both defined mainly at the early design stages. Fig. 2 relates travellers' needs and aspirations with the ICs discussed above and shows the proposed set of FRs to fulfil needs at the middle and top of the pyramid. These FRs are a translation of requirements fulfilled by high level DPs specified by the TFL Interchange [24]. Contrarily to what is found in the literature [3,25-26, to cite a few] which tends to show high level DPs biased by the zoning system of: 'Access \& Interchange zone', 'Facilities or Concourse zone' and 'Platform zone', the TFL Interchange [24] presents what are the primary spaces directly related to travellers' needs. The authors connect these DPs to travellers' needs by proposing the set of FRs listed in Fig 2.

'Speed' is a need directly related to travellers' routes; how fast to move as well as how far to move from any pair of origin - destination. As noted in $[5,13-18,26]$ speed is related to total traveling time which includes not only transportation time, waiting time and time required to walk from a specific point of the city to the terminal but also walking time inside the terminal building itself. Since there is no prescribed quantity associated to this portion of the total traveling time, the provision of short unobstructed traveller routes inside the building becomes an essential functional requirement (FR3).

'Easiness' is a need related to way-finding [13-18, 26-28], first in terms of efficiently assisting travellers in deciding where to go inside the terminal building (FR2). Second, it involves not only the provision of appropriate information but mainly how easy it is to make decisions related to travelling in general, such as buying ticket, passing control points, gathering information, etc. Specific types of spaces need to be provided for these decisions to take place and the shape, combination and location of these spaces are also an essential part of the building layout. 
'Comfort' and 'Experiences' are needs related to opportunities the building can offer to compensate for waiting time (FR1). These can be translated into, for instance, offering convenience shopping, food and refreshments, 'workstations', etc. [13-18, 26] Opportunities can also be exploited by non-travellers and business managers if terminals are configured as city hubs, neighbourhood 'hot spots', social and leisure places, etc. [26-28]. They are a FR in which multiple stakeholders' requirements need to be reconciled as, despite them being a place to enhance travellers' experience, terminals should also benefit citizens of the city and TIH neighbourhood, attract business investors, etc. to become sustainable.

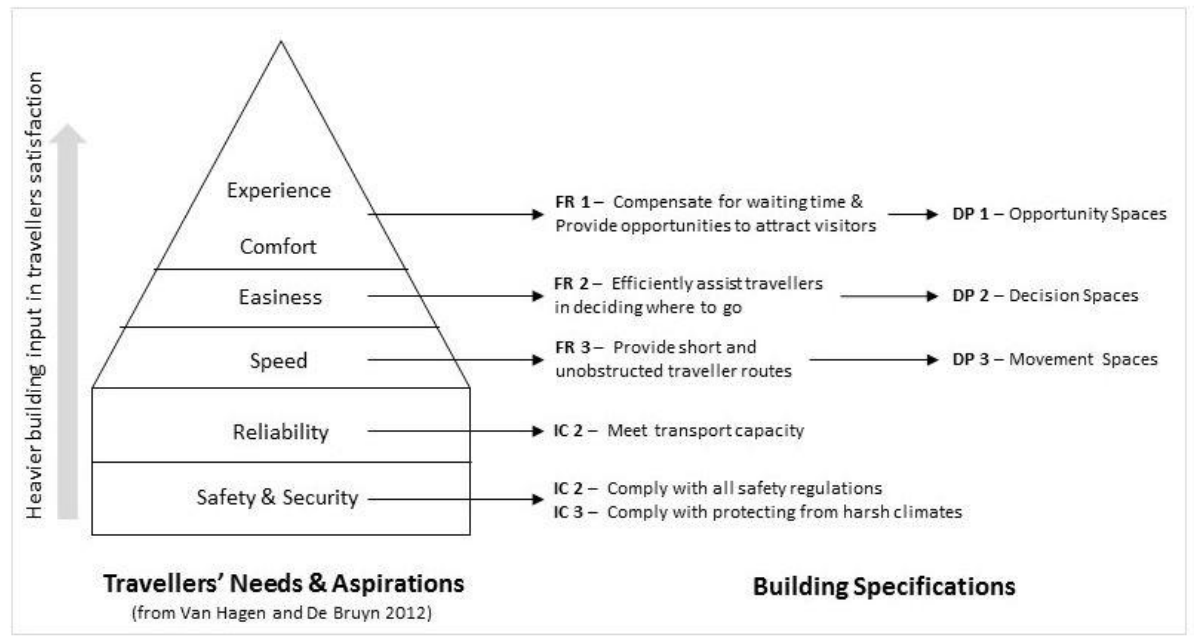

Fig. 2. Travellers' needs and aspirations with corresponding upper level building specification expressed as ICs, FRs and DPs.

High level DPs presented by the TFL Interchange [24] comprise the following three types of spaces to be managed inside TIH buildings: Movement Spaces (DP3), where travellers move from A to B within the terminal, Decision Spaces (DP2) where travellers' decisions take priority and Opportunity Spaces (DP1), which are actually all other spaces within the building which do not include movement or decision making and can therefore be used to enhance traveling experience as well as provide economic benefits to become sustainable. The TFL Interchange [24] affirms these are main spatial management principles to be used in the design of TIH buildings and "should be applied at brief development stage, and then considered throughout design development to evaluate design concepts against anticipated needs; and subsequently written into interchange facility management agreements to ensure design integrity is retained post-implementation" [24]. These high level FRs and their corresponding DPs seem to satisfy the First Axiom as the FRs are independent of each other and in principle could be independently distributed in space. The amount of content of the design at this stage is logical and minimal potentially satisfying also the Second Axiom. More importantly, these high-level DPs are abstract enough to promote layout diversity as they enable 
multiple combinations and can be adapted to multiple scale terminals, fulfilling well the different sizes of TIH building required by the Russian Arctic.

The multiplicity of combination enabled by these DPs can be better understood once they are decomposed into a set of subsequent FRs with their corresponding second level DPs as displayed in Fig. 3.

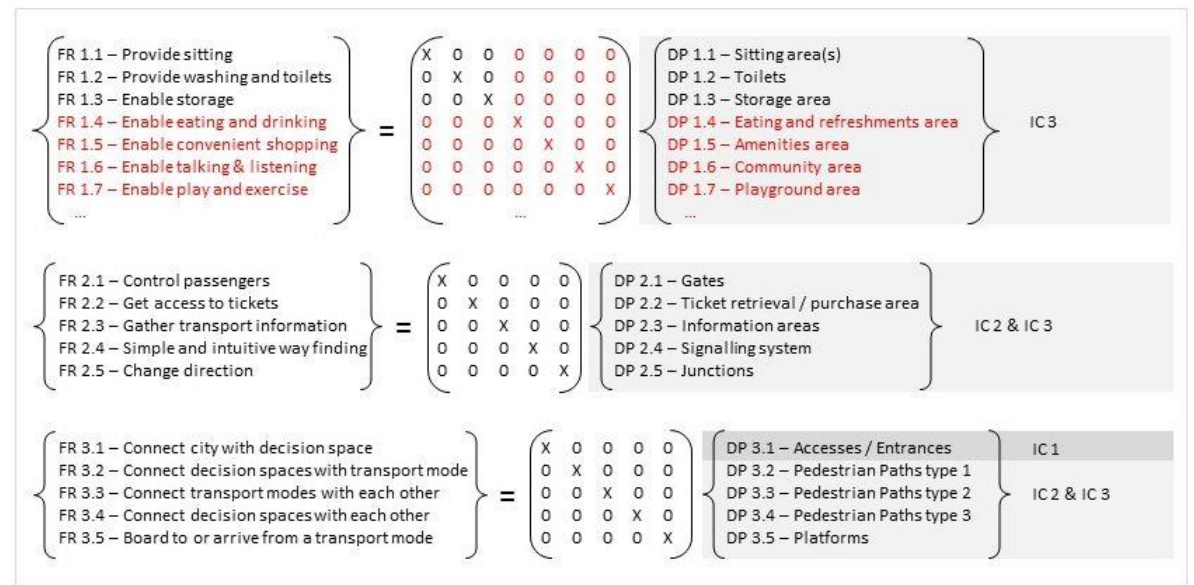

Fig. 3. Second level FRs and DPs with their corresponding design matrixes and constraints

Fig. 3 specifies second level FRs and DPs for the three different types of spaces, which need to be accommodated by a terminal building with their corresponding matrixes to study levels of independence among them. The very basic functions of Opportunity Spaces (DP 1) to compensate for travellers waiting time are expressed in black and include: an area to sit, washing and toilets and an area to store luggage. The additional functions represented in red show examples of enhancing travel experience during waiting times as well as providing opportunities to attract visitors and can include areas to eat and drink, shop, meet people, interact with the community, entertain children, etc. The list of FRs in this case will be context specific, i.e. it will depend on the scale and role the TIH will have in the urban environment, both locally and globally, which will establish its hierarchical importance in relation to the surrounding neighbourhood and/or city. NODES [27], provides a set of 5 different typologies of TIHs considering the presence of commercial areas within or around the terminals as well as the density of residential and mixed-use areas in the surroundings of the TIH and their respective impact on vehicular and pedestrian traffic congestion as well as parking needs. As this paper attempts to be generic enough to be adapted to different contexts, it leaves this matrix open for new FRs and DPs to be included accommodating different sizes of terminals. In theory, FRs related to Opportunity Spaces (DP 1) are considered independent from each other and will have to fulfil constraints related to regulatory frameworks for building in extreme climates (IC 3), if located within the building.

However, their size might have an impact in FR 3.1 and will be constrained by the insertion of the terminal in the city (IC 1). Further impact of FRs from DP1 and FRs 
from DP3 would need to be explored at a lower level and results from these explorations should be used to revisit level 2 FRs again, until a full set of independent FRs can be effectively reached in practice.

Functions of Decision Spaces (DP2) include: passing control lines to reach different types of transport modes, getting access to tickets, gathering information about transport operations as well as about where to go inside the terminal, and provide opportunities and information for passengers to change and find appropriate directions to take inside the building so they can reach its different spaces. These FRs are expressed as a set of DPs which are independent of each other (see Fig 3) with the corresponding design matrix to show they satisfy the first AD axiom. IC 3 and IC2 need to be applied to these DPs as these spaces need to respect strict thermal comfort constraints in order to be protected from the extreme climate and fulfil transport regulations and standards with regards to meeting capacity. Further decomposition of these second level DPs into second level FRs at an abstract level are still possible but they will be mainly related to interior design specifications (finishing) and the design of signalling, information and security systems, diverging from the scope of this paper which focuses mainly on TIH spatial layout. As in the case with decomposing DP1, there will be a stage in which the number and location of DPs 2.1, 2.2 and 2.5 will be affected and potentially dependent on DPs 1 and 3, however further decomposition is also necessary for these to be properly visualized and decoupling strategies to be applied.

Functions of Movement Spaces (DP3) include: entering the terminal building, walking from one decision space to another or to a transport mode, move between two different types of transport modes and boarding to or arriving from a transport mode. Three different types of constraints apply to these DPs: IC 1 which imposes restrictions in terms of how the building is inserted in the neighbourhood, IC 3 which imposes these spaces should all be protected from the harsh climate and IC 2 which establishes these spaces need to have adequate capacity to meet demand as imposed by the transport and traffic regulations. Note the separation between arrival and departure is not explicitly stated yet because these can be accommodated via specifying origin - destination for each type of pedestrian path. The level of independence between these FRs is expressed by the design matrix displayed in Fig 3. However, further decomposition of these DPs is needed to verify if assumed independence of FRs in level 2 holds true.

Fig 4, provides a level 3 set of FRs for DP 1.1, DP 3.1 and DP 3.2 to 3.4. FRs for sitting area (DP 1.1) have to provide enough capacity to accommodate a certain number of people waiting, including the elderly and those with disabilities which are mainly defined by constraints imposed by transport regulations (IC 2), and provide appropriate furniture for them. They need to be secure and 'naturally' surveyed by design (i.e. inhibit crime by allowing sufficient flow of people around them) and then meet a series of environmental quality criteria such as being well lit and ventilated, appropriately shaded and guaranteeing air temperatures within the comfort zone (from $18^{\circ} \mathrm{C}$ to $24^{\circ} \mathrm{C}$ ) as defined by the set of constraints referring to building in an extreme climate such as the Arctic (IC 3). Exploring subsequent DPs for these FRs involves assessing the number and positioning of these DPs in space, information which is context specific. 


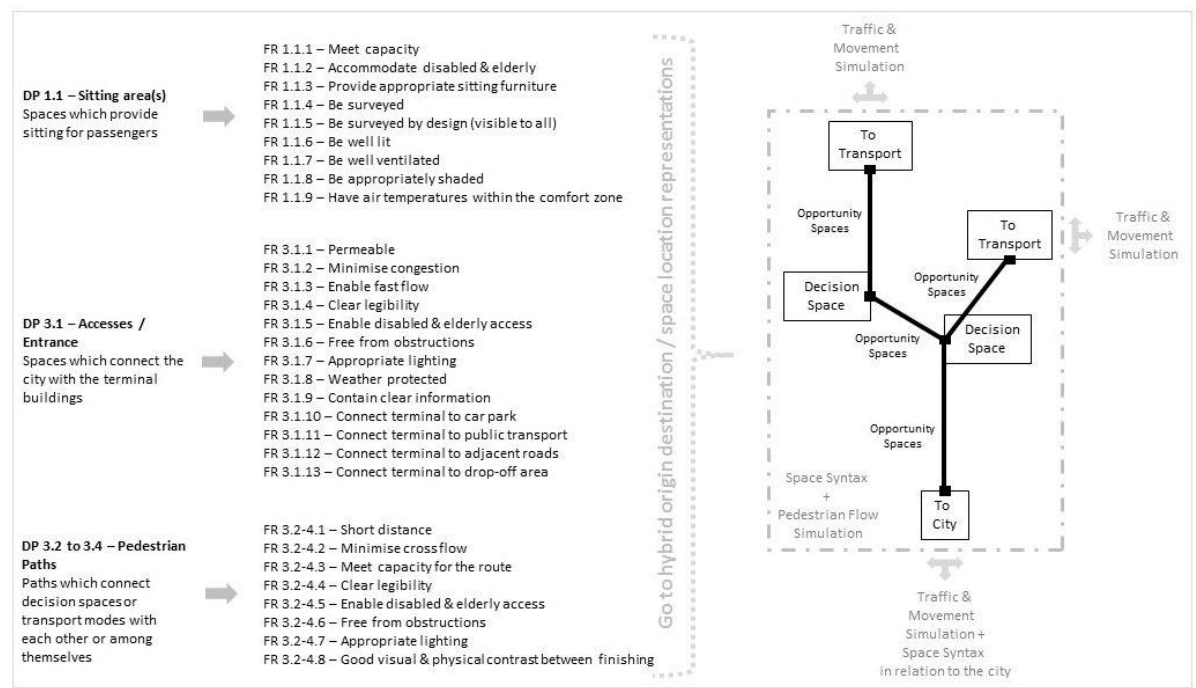

Fig. 4. Zooming into a set of DPs to illustrate the exploration of third level FRs in combination with a generic hybrid origin destination / space location representation.

Accesses / Entrances (DP 3.1) are the main points of connection between the terminal building and the city. Their positioning will determine how well the terminal integrates with the city at a neighbourhood scale and therefore should take into consideration local patterns of pedestrian movement, easy way finding for pedestrians and different types of vehicles as well as to and from movement routes for all transport modes reaching the building [26-28]. Thus, FRs for positioning Accesses / Entrances can be specified in terms of the quality of connections from terminal to: car park, public transport stops, drop-off area and points and areas characterized by high flows in adjacent open public spaces and roads. Beyond connections, FRs for DP 3.1 also need to: be permeable (i.e. integrating the surroundings and terminal as if they are a single space) and cognitively legible, minimize mobility congestion, enable fast flow of pedestrians to the terminal and circulation of vehicles around the terminal, be free from obstructions and universally accessible (disabled and elderly), have appropriate lighting and be weather protected (as imposed by IC 3). All these FRs will lead to context specific level 3 DPs as well as context specific constraints (IC 1).

DPs 3.2 to 3.4 need to be carefully examined so an adequate number of them is provided to meet the different terminal demands. This calls for diagrams to be produced so all potential origin - destination pairs of pedestrian movement within the terminal building are mapped to assess what needs to be given priority and therefore made shortest and with minimum crossing, what can be grouped or integrated with other path, what can be grouped and integrated with other type of space (e.g. be together with siting area, be together with eating and drinking area etc.). FRs at this level, include important requirements to satisfy travellers needs in relation to speed such as: short distance, minimum cross flow, segregation arrival / departure, adequate capacity, guaranteed disable access, lack of obstruction and clear spatial legibility. Again, exploring subsequent DPs for these FRs involves assessing the number and positioning of these DPs in space, 
information which is context specific. This is why in Fig 4 FRs are not followed by a set of third level DPs but by an abstract origin destination / space location diagram to represent context and be able to discuss how it can affect DPs and the construction of design matrices from this level down.

\section{$5 \quad$ Exploring the use of hybrid origin destination / space location representations and simulations to meet the Second Axiom, a discussion}

The aforementioned set of specifications suggest TIHs can be seen as buildings which basically host pedestrian flows with different types of origin-destination pairs. These origin-destination pairs are represented in a generic graph (Fig 4) as lines which connect different types of decisions spaces, access/entrance and transport boarding arrival points, surrounded by opportunity spaces, a combination of origin-destination graph with a space location diagram. This type of representation is essential to assess dependences at lower levels as it enables the visualization and exploration of different combinations of pedestrian flows and space distributions in site prior to their sizing and shaping. Hybrid representations are important to extract hierarchies of routes and spaces therefore providing important information for dependencies between FRs to be reassessed. In combination with matrices, they empower designers to make decisions in relation to moving towards a decoupled design and therefore in fulfilling the First Axiom, opening the possibility for matrices to be revisited at higher levels, corrected at this level and continued at lower levels, so the coherence of design specifications can be controlled. This process is not linear, it triggers a zigzag in the workflow through several iterations.

Besides that, hybrid representations are essential to aid in fulfilling the Second Axiom, as they provide information to simulate the performance of the overall design solution so probability ranges required by FRs and probability ranges provided by DPs can be adjusted to become compatible. They connect the design specification to simulation tools, in particular to those related to space connections, visual navigation, and pedestrian flow. Starting from the assumption that 'space is the machine' [29], there is a mutual dependency between built form and spatial navigation patterns, which could be explored both ways. The relation between the two is not one to one, meaning that the same flow pattern can be generated through different building layouts. In this sense the hybrid representation is a means to explore spatial configurations, without being tied to any given design outcome.

The fulfilling of many of the FRs related to positioning entrances / accesses and visual navigation inside the building can be investigated through the use of Space Syntax Theory [31], using combinations of Axial or Segment Analysis models, which translate mobility/street networks and internal building layouts in corresponding graph representations made of nodes (representing analysed elements) and connected edges (representing their topological links). Segment Analysis models can be complemented by fine-tuned Visibility Graph Analysis (VGA) models to explore the configurational qualities of the entrance spaces of a terminal building and how the TIH is connected 
with other public spaces and locations within a neighbourhood and a city; including the complexities implied in improving way-finding and increase cognitive ease in visually locating both the terminal and its accesses. Space Syntax models can also help clarifying the overlaps and dynamics between to-movement (i.e. movement to the TIH as a destination from all other points in the city), and through-movement flows (i.e. movement passing through on shortest routes from every origin to every destination). To this end, a multidimensional study of street networks must be conducted both at a local (the immediate surroundings) and at a global level (the city as a whole) as the "urban space is locally metric but globally topo-geometric" [32]. As suggested by [27-31], when properly deployed Space Syntax offers useful guidance for integrating the design of TIH and surrounding public open spaces while increasing travellers' safety though natural surveillance and co-awareness.

The success of fulfilling FRs related to the positioning of entrances / accesses as well as the positioning of platforms to different transport modes, should also be investigated through Traffic and Movement simulations. Micro-simulations, which include car following models, can be used, for instance, to examine points of congestions around the terminal building and assess delays and their potential impact in overall travel time. They can also be used to study lanes and intersection geometry assisting in assessing how well integrated the terminal is with the city's infrastructure system as well as how good is its accessibility.

Besides that, the different types of pedestrian paths inside the building connecting decision spaces with transport modes, different types of transport modes with each other and different types of decision spaces with each other can also be assessed using pedestrian flow simulations in combination with Space Syntax. The domain of pedestrian modelling is widely explored to assess station performance in relation to a series of parameters which impact on health and safety as well as in total travel time. They are used to size paths, test how their length, positioning and connections affect total travel time, test the impact of obstructions in disrupting pedestrian flow, test and size control gates and platform area, etc. They enable density maps to be generated showing waiting times, emergency evacuation routes, clearing times, journey times in different routes, etc. [34]. The authors discuss in more detail the issue of pedestrian flow in the design of TIH in another paper also submitted to this conference [35].

Space Syntax can contribute to assess TIHs' interior spatial intelligibility, visual relationships and visual-control among spaces or spatial enclosure, signals positioning as well as the perceived socio-spatial qualities of opportunity spaces. Given that past research has found a correlation between the Integration index and density of economic activities in cities [33], the retrieval of Space Syntax metrics could in fact effectively inform the location lorganisation of opportunity spaces within terminal buildings.

Since results from simulation related to traffic and movement as well as pedestrian flow are expressed as probabilities, they can be used to test if design specifications meet the Second Axiom which states that in order to minimize information, "the design must be able to accommodate large variations in design parameters (...) and yet still satisfy the functional requirements" [20]. This association of probability ranges with design specifications and simulations moves the problem away from analysis multi-criteria and establishes a different design paradigm. 


\section{Conclusions and future work}

The way terminals are operated, the differences in preferences among its users and the way they should respond to the environment and the different functions they host go beyond 'design by drawing'. The fact that the context chosen for this discussion was the Russian Arctic also introduced extra challenges to the design problem. Main challenges involved: (i) the need for space enclosures and temperature controls in almost all types of spaces, (ii) the need for design specifications which can cope with a high variety in building size (from a couple of platforms to large multimodal terminals), and (iii) a design system which opens the possibility of properly taking into account the impact of variations in population fluctuations and the consequences of these on requiring reducing or expanding terminal capacity.

Instead of opting for analysis multi-criteria which is always controversial in terms of justifying the different weights attributed to each different objective to be met, the authors proposed the use of AD to organize information from the literature in relation to designing the layout of TIH. The AD structure helps to map design specifications, control them through the application of the First Axiom, and assess them using the Second Axiom. It is therefore considered a robust method to reduce gaps between what a designer knows about a problem, what is the proposal to solve it and what solution is actually delivered.

Going deep into design specifications and applying the First Axiom reduced the amount of complexity initially associated with layout design by simplifying its rationale to three different types of spaces: control spaces, movement spaces and opportunity spaces. This rationale is adaptable to different building sizes which not only fits into the Arctic context but can also be transferred to many other places. The use of AD also changed the approach to design priorities, from the simple fulfilling multiple assessment criteria to the search for a clear hierarchy of pedestrian flows to be evaluated using different types of computer simulation tools. Beyond that, the idea of using the Second Axiom to coordinate this group of simulation tools and relate them to design specifications, opens the possibility for uncertainty to be inserted into the design problem from its very beginning. It also changes the way designers are supposed to deal with uncertainty by not having it exclusively in the solution domain anymore but also bringing it to the design specification domain. If, on one hand, this can break designers free from the paradigm of 'classic' design optimization, it brings, on the other hand, a series of new challenges to the design process such as for instance how can probability ranges (tolerance values) be assigned to DPs which are mainly defined as spaces or spatial properties (either at a building level of neighbourhood scale level)?

The implementation of AD in writing design specification for TIHs is not supposed to end with this paper. Beyond, future work on fully exploring the Second Axiom, should also include developing and overlaying design specifications for different stakeholders and dealing with potential conflicts arising from it. Furthermore, it should be tested in different contexts and in practice. 


\section{References}

1. Monzon, A.: Innovative design and operation of new or upgraded efficient urban transport interchanges. In: Monzon, A. (eds.) City-HUB. Project Report. European Commission, Seventh Framework Cooperation Work Programme (2015).

2. Yatskiv, I., Nathaniel, E., Richter, K.: Special issue introduction: Research in sustainable transport interchanges. Transport and Telecommunication 9(3), 179-182 (2018).

3. Jia, J. and Fang, Y.: Underground space development in comprehensive transport hubs in China. Procedia Engineering 165, pp. 404-417 (2016).

4. Li, L., Ren, H., Zhao, S., Duan, Z., Zhang, Y., Zhang, A.: Two dimensional accessibility analysis of metro stations in Xi' an, China. Transportation Research Part A 106, pp.414-426 (2017).

5. Liu, R., Chen, Y., Wu, J., Xu, T., Gao, L., Zhao, X.: Mapping spatial accessibility of public transportation network in an urban area - A case study on Shanghai Hongqiao Transportation. Transportation Research Part D 59, pp.478-495 (2018).

6. Dunichkin, I. V.: Transport interchange hubs under the conditions of the Far North. In: Murgul, V. and Popovic, Z. (eds.) INTERNATIONAL SCIENTIFIC CONFERENCE ENERGY MANAGEMENT OF MUNICIPAL TRANSPORTATION FACILITIES AND TRANSPORT EMMFT, pp. 446-452. Advances in Intelligent Systems and Computing 692, Khabarovsk (2017).

7. HERMES - High efficient and reliable arrangements for crossmodal transport, https://cordis.europa.eu/project/rcn/93149/reporting/en, last accessed 2019/02/10

8. City-HUB, http://www.cityhub.imet.gr/, last accessed 2019/02/10

9. NODES Interchanges - New tools for designing and operation of urban transport interchanges, http://www.nodes-interchanges.eu/, last accessed 2019/02/10

10. Alliance - Enhancing excellence and innovation capacity in sustainable transport interchanges, http://alliance-project.eu/, last accessed 2019/02/10

11. VitalNodes - Building a lasting expert network that delivers evidence-based recommendations for Vital Urban Nodes along TEN-T Corridors, https://cordis.europa.eu/project/rcn/212872/factsheet/en, Last accessed 2019/02/10

12. Zamyatina, N., Goncharov, R., Population mobility and the contrasts between cities in the Russian Arctic and their southern Russian counterparts. Area Development and Policy 3(3) 293-308. (2018)

13. Hickman, R., Chen, C.L., Chow, A., Saxena, S.: Improving interchanges in China: the experiential phenomenon. Journal of Transport Geopgraphy, 42. pp. 175-186 (2015).

14. Hernandez, S., Monzon, A., Ona, R.: Urban transport interchanges: A methodology for evaluating perceived quality. Transportation Research Part A 84, pp. 31-43 (2016).

15. Tsami, M., Adamos, G., Natrhanail, E., Budilovich, E., Yatskiv, I., Magginas, V.: A decision tree approach for achieving high customer satisfaction at urban interchanges. Transport and Telecommunication, 19(3), 194-202 (2018).

16. Monzon, A., Alonso, A., Lopez-Lambas, M.: Joint analysis of intermodal long distance-last mile trips using urban interchanges in EU cities. Transportation Research Procedia 27, pp. 1074-1079 (2017).

17. Bryniarska, S., Zakowska, L.: Multi-Criteria evaluation of public transport interchanges. Transportation Research Procedia 24, pp. 25-32 (2017).

18. Van Hagen, M., de Bruyn, M.: The ten commandments of how to become a customer-driven railway operator. EUROPEAN TRANSPORT CONFERENCE, pp. 1-19. Association for European Transport AET, Glasgow (2012). 
19. Marchesi, M. and Matt, D. T.: Application of Axiomatic Design to the design of the built environment: A literature review. In: Farid, A.M. and Suh, N.P. (eds.) Axiomatic Design in large systems. pp. 151-174. Springer International Publishing Switzerland (2016)

20. Suh, N. P.: Axiomatic Design: Advances and Applications. Oxford University Press, New York (2001).

21. Pastor, J. B. R., Benavides, E. M.: Axiomatic Design of an airport passenger terminal. In: Thompson, M. K. (eds.) PROCEEDINGS OF THE $6^{\mathrm{TH}}$ INTERNATIONAL CONFERENCE ON AXIOMATIC DESIGN, ICAD, pp.95- 102. Korean Advanced Institute of Science and Technology (KAIST), Daejon (2011).

22. N. I. Shiklomanov, D. A. Streletskiy, V. I. Grebenets, L. Suter. Conquering the permafrost: Urban infrastructure development in Norilsk, Russia. Polar Geography 40(4), pp 273-290 (2017).

23. Russian Transport Regulations SNIP 2.07.01-89

24. Transport for London (TfL) Interchange. Interchange best practice guidelines 2009. Quick reference guide. Homepage: www.tfl.gov.uk/interchange, last accessed 28/01/2019.

25. Monzon, A., Hernandez, S., Di Ciommo, F.: Efficient urban interchanges: the city-HUB model. Transportation Research Procedia 14, pp. 1124-1133 (2016).

26. Booth, R.: D3.3.1 Identification and specification of the key areas of interchange design. In: Hoogendoom, C. (eds.) NODES 'New tOols for Design and OpEration of Urban Transport InterchangeS'. Project Report. European Commission, DG Research and Innovation (2015).

27. Aldecoa, J.: D3.2.1 Mobility analysis and urban planning for interchanges. In: Hoogendoom, C. (eds.) NODES 'New tOols for Design and OpEration of Urban Transport InterchangeS'. Project Report. European Commission, DG Research and Innovation (2015).

28. Pastor, A.G., Pinedo, J.C.: D3.2.2 Urban planning and development at a close-up level. In: Hoogendoom, C. (eds.) NODES 'New tOols for Design and OpEration of Urban Transport InterchangeS'. Project Report. European Commission, DG Research and Innovation (2015).

29. Hillier, B., Space is the Machine: A Configurational Theory of Architecture. Cambridge University Press, Cambridge, UK. (1996)

30. Dauden, F. J. L., Carpio-Pinedo, J., Garcia-Pastor, A.: Transport interchange and local urban environment integration. Procedia - Social and Behavioural Sciences 160, pp. 251-223 (2014).

31. Hillier, B., Hanson, J., The Social Logic of Space by Bill Hillier [WWW Document]. Cambridge Core. https://doi.org/10.1017/CBO9780511597237 (1984)

32. Hillier, B., Turner, A., Yang, T., Park, H.-T., Metric And Topo-Geometric Properties Of Urban Street Networks: Some convergences, divergences and new results. The Journal of Space Syntax 1, 279. (2010)

33. Hillier, B., Cities as movement economies, in: Urban Design International, 1: 49-60. (1996).

34. Brookes, I, Greenwood, R., Wilson, T., Yariv, B.: Station Design Principles for Network Rail. Safety Technical \& Engineering Guidance. Document n. BLDG-SP80-002. Network Rail, UK (2015)

35. Dunichkin, I.V., Bleil de Souza, C., Bogachev, K., Pezzica, C. Exploring specific features of Transport Interchange Hubs (TIH)design, taking into account the climatic conditions of the Russian Arctic. International Conference on Computational Science and its Applications. Under review (2019). 